\title{
RESEARCH
}

Open Access

\section{The impact of caring for dying patients in intensive care units on a physician's personhood: a systematic scoping review}

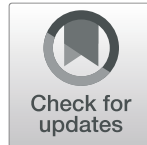

Joshua Tze Yin Kuek ${ }^{1,2}$, Lisa Xin Ling Ngiam², Nur Haidah Ahmad Kamal ${ }^{1,2}$, Jeng Long Chia ${ }^{1,2}$, Natalie Pei Xin Chan ${ }^{1,2}$, Ahmad Bin Hanifah Marican Abdurrahman ${ }^{1,2}$, Chong Yao Ho ${ }^{1,2}$, Lorraine Hui En Tan ${ }^{1,2}$, Jun Leng Goh ${ }^{1,2}$, Michelle Shi Qing Khoo ${ }^{1,2}$, Yun Ting Ong ${ }^{1,2}$, Min Chiam³ ${ }^{3}$ Annelissa Mien Chew Chin ${ }^{4}$, Stephen Mason ${ }^{5}$ and Lalit Kumar Radha Krishna ${ }^{1,2,3,5,6,7,8^{*}}$ (D)

\begin{abstract}
Background: Supporting physicians in Intensive Care Units (ICU)s as they face dying patients at unprecedented levels due to the COVID-19 pandemic is critical. Amidst a dearth of such data and guided by evidence that nurses in ICUs experience personal, professional and existential issues in similar conditions, a systematic scoping review (SSR) is proposed to evaluate prevailing accounts of physicians facing dying patients in ICUs through the lens of Personhood. Such data would enhance understanding and guide the provision of better support for ICU physicians.

Methods: An SSR adopts the Systematic Evidenced Based Approach (SEBA) to map prevailing accounts of caring for dying patients in ICUs. To enhance the transparency and reproducibility of this process, concurrent and independent use of tabulated summaries, thematic analysis and directed content analysis (Split Approach) is adopted.

Results: Eight thousand three hundred fifty-eight abstracts were reviewed from four databases, 474 full-text articles were evaluated, 58 articles were included, and the Split Approach revealed six categories/themes centered around the Innate, Individual, Relational and Societal Rings of Personhood, conflicts in providing end of life care and coping mechanisms employed.

Conclusion: This SSR suggests that caring for dying patients in ICU impacts how physicians view their personhood. To resolve conflicts within individual concepts of personhood, physicians use prioritization, reframing and rely on accessible, personalized support from colleagues to steer coping strategies. An adapted form of the Ring Theory of Personhood is proposed to direct timely personalized, appropriate and holistic support.
\end{abstract}

Keywords: Intensive care unit (ICU), Physicians, Death and dying, Ring theory of personhood (RToP), Personhood, Resilience

\footnotetext{
*Correspondence: lalit.radha-krishna@liverpool.ac.uk

${ }^{1}$ Yong Loo Lin School of Medicine, National University of Singapore,

Singapore, Singapore

${ }^{2}$ Division of Supportive and Palliative Care, National Cancer Centre Singapore, Singapore, Singapore

Full list of author information is available at the end of the article
}

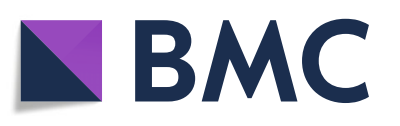

(- The Author(s). 2020 Open Access This article is licensed under a Creative Commons Attribution 4.0 International License, which permits use, sharing, adaptation, distribution and reproduction in any medium or format, as long as you give appropriate credit to the original author(s) and the source, provide a link to the Creative Commons licence, and indicate if changes were made. The images or other third party material in this article are included in the article's Creative Commons licence, unless indicated otherwise in a credit line to the material. If material is not included in the article's Creative Commons licence and your intended use is not permitted by statutory regulation or exceeds the permitted use, you will need to obtain permission directly from the copyright holder. To view a copy of this licence, visit http://creativecommons.org/licenses/by/4.0/. The Creative Commons Public Domain Dedication waiver (http://creativecommons.org/publicdomain/zero/1.0/) applies to the data made available in this article, unless otherwise stated in a credit line to the data. 


\section{Background}

The COVID-19 pandemic has overwhelmed healthcare systems and has physicians facing unprecedented levels of death and dying in Intensive Care Units (ICU)s [1, 2]. Received knowledge suggests that these experiences impact the emotional, psychological and physical wellbeing of ICU physicians, though there is little understanding of how these complex, frequently and closely related issues arise, much less are supported [3-5]. This dearth of data has raised concerns about how ICU physicians cope amidst the COVID-19 pandemic.

In the face of data suggesting that nurses in ICU report similar personal, professional and existential issues in comparable circumstances [6-12], a systematic scoping review (SSR) was proposed to study accounts on the impact of caring for dying patients on physicians in the adult ICU in the extent literature. Better understanding of how ICU physicians cope with death and dying could inform efforts to support them during the pandemic and beyond.

\section{Methods}

An SSR is proposed to map current data on the subject. To enhance its reproducibility and transparency, the Systematic Evidenced Based Approach (SEBA) was used to guide the SSR (henceforth SSR in SEBA). The SSR in SEBA's constructivist perspective captures contextual factors whilst a relativist lens facilitates the inculcation of diverse experiences and perspectives of ICU physicians caring for dying patients [13-16]. The research team was supported by medical librarians from the Yong Loo Lin School of Medicine (YLLSoM) at the National University of Singapore and National Cancer Centre Singapore (NCCS), and local experts and clinicians at NCCS, the Palliative Care Institute Liverpool, YLLSoM and Duke-NUS Medical School (henceforth the expert team) to enhance the reproducibility, transparency and trustworthiness of this analysis. The principles of interpretivist analysis were employed in the 5 stages of SEBA highlighted in Fig. 1.

\section{Stage 1 of SEBA: systematic approach Determining the title and research question}

To ensure a systematic approach, the research and expert teams established the goals of the SSR and the population, context and concept (PCC) to be evaluated. The primary research question was determined to be: "what is known of how physicians contend with death and dying in the adult ICU setting?" and the secondary questions: "what is the impact of caring for dying patients on physicians in the adult ICU?" and "how do these effects manifest?"

\section{Inclusion criteria}

A PICOS format was adopted to guide the research process as shown in Table $1[17,18]$.

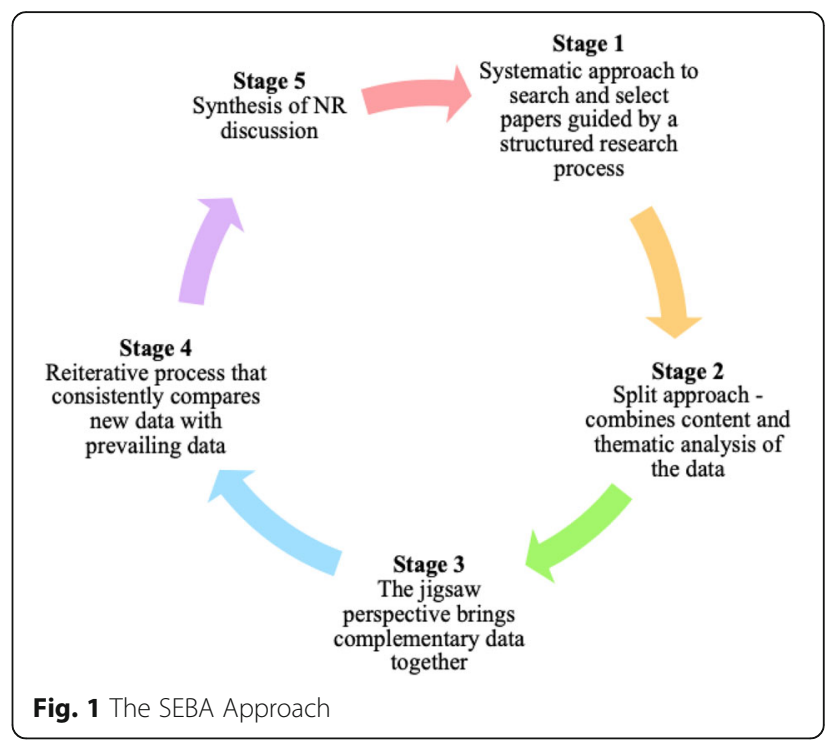

\section{Searching}

The 11 members of the research team carried out independent searches of four bibliographic databases (PubMed, Embase, CINAHL, and PSYCINFO) for articles published between 1st January 1990 and 31st December 2019. The searches were carried out between 13th February 2020 and 24th April 2020. The PubMed search strategy may be found in Additional file 1.

Each member of the research team independently sieved through all titles and abstracts from the individual searches of the four databases and created their own lists of titles to be reviewed. Comparing these individual lists via online meetings, the teams used 'negotiated consensual validation' to achieve consensus on the final list of titles to be reviewed [19].

The research team then independently reviewed each of the full-text articles from this final list, created individual lists of articles to be included, discussed them online and achieved a consensus on the final list of full-text articles to be included in the SSR. The results of this process are outlined below.

\section{Results}

Eight thousand three hundred fifty-eight abstracts were identified from four databases, 7973 articles were reviewed, and 58 articles were included as shown in Fig. 2.

\section{Stage 2 of SEBA: Split approach}

Three teams of at least three researchers independently reviewed the 58 included full-text articles.

The first team summarized and tabulated them as recommended by Wong, Greenhalgh [20]'s RAMESES publication standards: meta-narrative reviews and Popay, Roberts [21]'s "Guidance on the conduct of narrative 
Table 1 PICOS, inclusion criteria and exclusion criteria applied to literature search

\begin{tabular}{|c|c|c|}
\hline & Inclusion criteria & Exclusion criteria \\
\hline \multirow[t]{9}{*}{ Population } & \multirow[t]{9}{*}{ • Physicians } & - Main focus on other health professionals such as: \\
\hline & & ${ }^{\circ}$ Nurses \\
\hline & & ${ }^{\circ}$ Allied health workers \\
\hline & & ${ }^{\circ}$ Healthcare support staff \\
\hline & & - Main focus on patients, caregiver, family or friends \\
\hline & & - Students from health professions such as: \\
\hline & & ${ }^{\circ}$ Medical students \\
\hline & & - Nursing students \\
\hline & & ${ }^{\circ}$ Allied health students \\
\hline \multirow[t]{8}{*}{ Intervention / Exposure } & \multirow{8}{*}{$\begin{array}{l}\text { - Being involved in the care of dying patients in } \\
\text { the adult ICU }\end{array}$} & - No involvement in care of dying patients \\
\hline & & $\begin{array}{l}\text { ' No clearly defined patient care experience } \\
\text { (e.g. study just explores attitudes to death/ palliative care) }\end{array}$ \\
\hline & & $\begin{array}{l}\text { - Patient population not dying patients } \\
\text { (incl. "geriatrics", patients without specification that they are dying) }\end{array}$ \\
\hline & & - Physician assisted suicide/ medical assistance in death/ suicide \\
\hline & & - Personal experience of death of family/ friend \\
\hline & & - Non-adult ICUs such as: \\
\hline & & - Paediatric ICUs \\
\hline & & ${ }^{\circ}$ Neonatal ICUs \\
\hline \multicolumn{3}{|l|}{ Comparison } \\
\hline Outcome measures & $\begin{array}{l}\text { - Impact on doctors } \\
\text { 'Emotional } \\
\text { - Psychological } \\
\text { 'Behavioural } \\
\text { 'Physical }\end{array}$ & \\
\hline Study design & $\begin{array}{l}\text { - English language } \\
\text { - Time of publication between } 1990 \text { and } 2019 \\
\text { - No restriction on study design (qualitative, } \\
\text { quantitative, mixed) } \\
\text { - No restriction on geographical location of } \\
\text { study or publication }\end{array}$ & $\begin{array}{l}\text { - Grey Literature, electronic and print information not controlled by } \\
\text { commercial publishing } \\
\text { - Narrative literature reviews without methodology } \\
\text { - Case reports and series, commentaries, editorials, and perspectives } \\
\text { - Non-English publications without English translation } \\
\text { - Unable to retrieve full article }\end{array}$ \\
\hline
\end{tabular}

synthesis in systematic reviews". The tabulated summaries ensure that key points of the articles are not lost (Additional file 2). The team also evaluated the quality of quantitative and qualitative studies included in this review using the Medical Education Research Study Quality Instrument (MERSQI) [22] and the Consolidated Criteria for Reporting Qualitative Studies (COREQ) [23].

Concurrently, the second team independently analyzed the 58 articles using Braun and Clarke [24]'s approach to thematic analysis while the third team adopted Hsieh and Shannon [25]'s approach to directed content analysis. Enhancing the reliability of the analyses, concurrent thematic and directed content analysis is a key feature of the 'Split Approach'.

The narrative produced by consolidating the tabulated summaries, themes and categories was guided by the Best Evidence Medical Education (BEME) Collaboration guide [26] and the STORIES (STructured apprOach to the Reporting In healthcare education of Evidence Synthesis) statement [27].

\section{Themes identified using thematic analysis}

The themes identified were the impact of caring for the dying on one's self; relationships; interactions; conflicts in providing end of life (EoL) care and the coping strategies employed. Elements of each of these themes are featured in Table 2.

\section{Categories identified using directed content analysis}

Positing that ICU physicians will be as similarly affected as their nursing colleagues [6-12] and will likely face changes in the manner that they view themselves, their relationships with family, friends, patients, and their changing roles [6-12], the research and expert teams adopted Radha Krishna and Alsuwaigh [28]'s Ring Theory of Personhood (RToP), an evidence-based framework to 


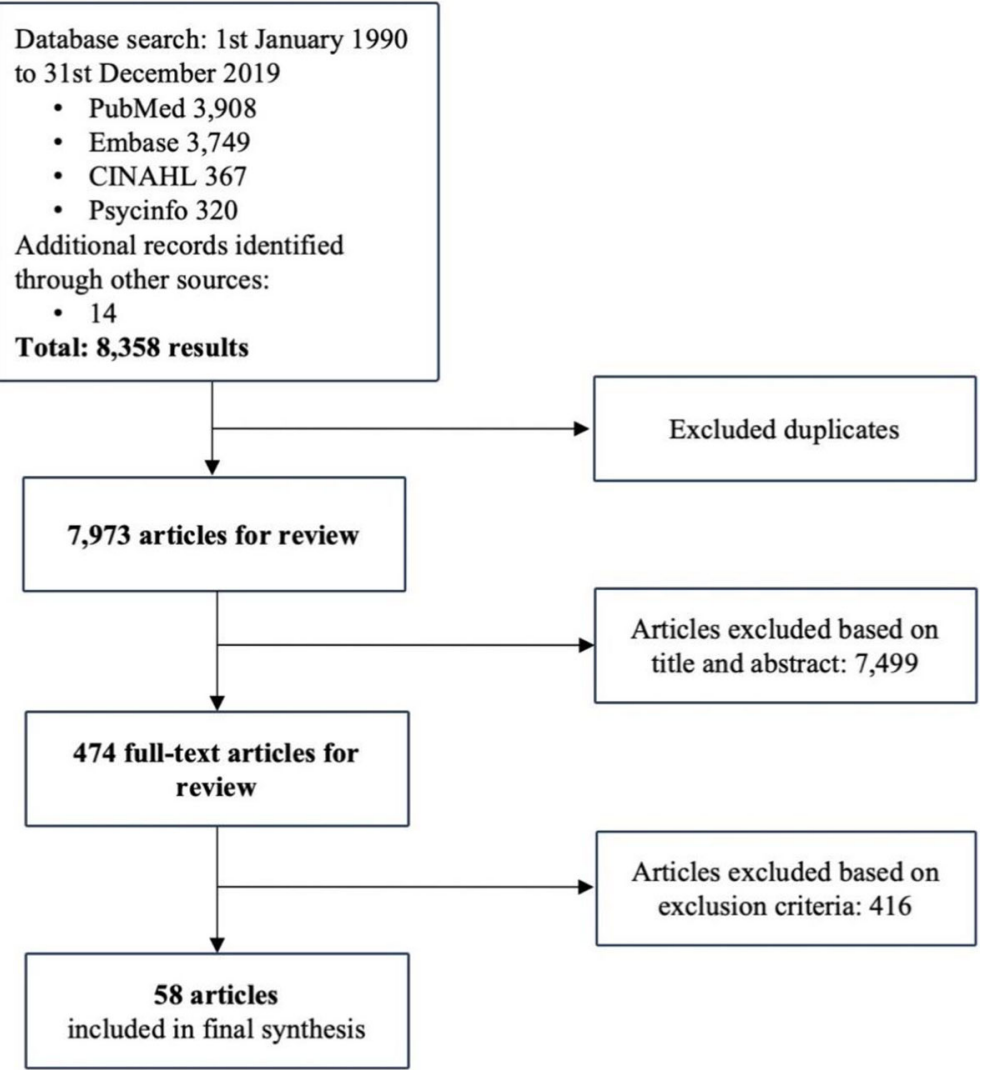

Fig. 2 PRISMA Flow Chart

studying concepts of personhood - or "what makes you, you" to study the effects of caring for dying patients in ICU on physicians.

The RToP consists of four aspects of personhood which form a complex interplay with one another as shown in Fig. 3.

At the core of the Ring Theory is the Innate Ring. Krishna and Alsuwaigh (2015) defined the Innate Ring as being present for all humans by virtue of their Divine connections and/or their genetic propensity to being a human. This Innate Ring serves a critical role in ensuring that the individual is treated with the dignity and care afforded all human beings until death.

The Individual Ring comprises the particular characteristics of the individual, such as their values, beliefs, and personality, as well as their conscious functions such as their abilities, thoughts, emotions and actions.

The Relational Ring consists of important personal relationships to the individual, such as ones with loved ones and close friends. These ties are determined by the person themselves and can change over time.

The Societal Ring is the outermost ring that consists of less intimate relationships as well as societal, religious, professional and legal expectations set out in the individual's society to guide and police conduct.
Adopting the RToP given its use in the end of life setting, the research team drew codes and categories from its description. Deductive category application [29] revealed four categories corresponding to the impact on the Innate, Individual, Relational and Societal Rings of Personhood Further elaboration is offered in Table 2 (without references) and in Additional file 3 (with references).

\section{Stage 3 of SEBA: funneling}

A funneling approach was adopted to streamline results from the three aspects of the Split Approach. It sees data compared and combined to reduce overlap and repetition whilst retaining a holistic perspective of the data. Critical aspects are identified and a jigsaw perspective is applied to put together 'complementary pieces' to generate a comprehensive map of the prevailing data on the impact of caring for dying patients on ICU physicians.

\section{Stage 4 of SEBA: the reiterative process}

Themes/categories identified were discussed with the expert team and with other local educationalists and clinicians. This process saw the research and the expert teams identify three themes (the impact of caring for dying patients on one's self, relationships and interactions) to be similar to categories identified from the 
Table 2 Summary of Thematic Analysis and Direct Content Analysis

Themes and examples
Theme 1: Self
Emotion
1. Internal conflict
2. Management of own expectations
3. Confrontation with own mortality
4. Apprehension/Distress
5. Fear due to unintentional transference to own family members
6. Satisfaction in providing end-of-life care

\section{Thoughts}

1. Doubt

2. Perception of emotional involvement

3. Professional responsibility

4. Death of a patient perceived to be a personal failure

5. Death of a patient not perceived to be a personal failure

6. Intervention as prolonging suffering for patients

7. Intervention as prolonging suffering for patient's family

8. Withdrawal of treatment as life-shortening

9. Decision between active treatment or palliative intention

10. Perception that nurses do not grasp the complexity of end-of-life decision making

11. Motivated to improve communication skills

12. Perception of intensive care unit as not conducive for palliative care discussions

\section{Behavior}

1. Impaired ability to make decisions

2. Impaired ability to communicate

3. Emotional detachment

4. Difficulty and discomfort when broaching topic of death to patients

5. Attempts to avoid discussion of death in general

6. Fear of litigation leading to defensive practice

7. Adherence to decisions despite potential legal kickback

8. Personal, patient, institutional and societal factors affecting decision making

9. Poor translation of spiritual ideas to goals of care

\section{Theme 2: Relationships}

\section{Physician's family}

1. Fear due to unintentional transference to own family members

\section{Theme 3: Interactions}

\section{Patients}

1. Challenges during end-of-life communication

2. Managing expectations of patients

3. Inspiring interactions with patients

\section{Categories and Example}

Category 1: Innate Ring

Perception of life and death

1. Confrontation with own mortality

2. Conception of a good death impacting end-of-life care

3. One has a right to die

\section{Category 2: Individual Ring}

Ability to make sense of things

1. Impact ability to make decisions

\section{Abilities to communicate}

1. Loss of ability to communicate and relate to patients

2. Improvement in communication skills

\section{Abilities to express feelings}

1. Emotional detachment

2. Emotion connection

\section{Acquired ability}

1. Lack of knowledge about end-of-life

2. Inadequate opportunities for end-of-life care training

3. Doubt and lack of confidence in clinical skills

4. Testing of practical skills such as treatment withdrawal techniques

5. Acquisition of new skills with experience

Beliefs

Personal Beliefs

1. Dilemmas about the balancing of opposing values

2. Personal beliefs reflected in end-of-life practices and communication

Exposed to Ethical dilemmas such as:

1. Differences in ethical opinion surrounding treatment withholding and withdrawal

2. Futile treatment

3. Lack of advanced directives and families' aggressive care requests causing moral distress

Religious views

1. Influenced end-of-life discussion and decision making

2. Did not influence end-of-life practices

\section{Perceived role as a doctor}

1. Perceived duty to prolong life causing moral distress

2. Uncertainty about role in end-of-life discussions resulting in no/late end-of-life discussion

3. Paternalistic approach to decisionmaking

4. Satisfaction upon reconciling dual role of saving lives and managing death well

\section{Category 3: Relational Ring}

Family

1. Fear due to unintentional transference to own family 
Table 2 Summary of Thematic Analysis and Direct Content Analysis (Continued)

Themes and examples

\section{Patient's Family}

1. Experiencing conflict with patient's family

2. Effects of conflict on the relationship

3. Family's concern for patient's possible pain and distress

4. Managing expectations of patient's family

5. Family's distress after end-of-life care discussion

6. Empowering interactions with patient's family

7. Factors affecting communication

8. Creation of soft landing when informing patient's family about death

9. Perception of intensive care unit as not conducive for palliative care discussions

\section{Nurses \& ICU Team}

1. Conflict between physician and intensive care unit nurses

2. Perception that nurses do not grasp the complexity of end-of-life decision making

3. Receiving support from other intensive care unit physicians in managing end-of-life decisions

\section{Physicians from other specialties}

1. Challenges with interactions

2. Lack of understanding of one another's role

Theme 4: Conflicts in providing end-of-life care

Societal Culture

1. Societal culture impacting decision making

2. Stigma associated with death or talking about death

\section{Workplace Culture}

1. Shapes the way doctors view death

\section{ICU Environment}

1. Suitability for palliative care teaching

2. Intensive care unit as an inappropriate place to die

\section{Legal environment}

1. Uncertainty with regards to legal implication of end-of-life practice

\section{Theme 5: Coping strategies}

\section{Personal strategies}

1. Effective communication to strengthen decision making position

2. Gaining confidence through experience and with end-of-life discussions

3. Taking breaks from the intensive care unit or practicing on other sites

\section{Categories and Example}

members

\section{Category 4: Societal Ring}

Physical environment

1. Availability of resources in different countries influencing end-of-life care

\section{Cultural environment}

1. Physician's end-of-life care attitudes, behaviors and decisions privy to cultural norms

2. Death and dying perceived as a "taboo" topic in certain cultures

3. Need for end-of-life care to be sensitive to different cultures encountered

4. Workplace culture impacting attitudes and practices

\section{Societal expectations}

1. Societal expectations promoting survival and death prevention leading to negative perception of treatment withdrawal as the taking of a patient's life, affecting physician's end-of-life decision making

\section{Legal standards}

1. Fear of litigation leading to defensive practice

2. Adherence to decisions despite potential legal kickback

3. Unclear laws surrounding end-of-life practices breeding legal uncertainty

\section{Professional Relationships}

1. Conflict relating to end-of-life decisions with patient's family and other healthcare professionals

2. Positive professional relationships

\section{Professional standards}

1. Professional expectation that doctors should not cause death or harm to patients

2. Responsibility of treatment withdrawal decision going against physician's perceived professional standards 
Table 2 Summary of Thematic Analysis and Direct Content Analysis (Continued)

\begin{tabular}{l} 
Themes and examples \\
\hline Strategies with patients \\
1. Collaboration with patients to reduce moral burden of decision \\
making \\
Strategies with patient's family \\
1. Creation of soft landing when informing patient's family about \\
death \\
2. Collaboration with patient's family to reduce moral burden of \\
decision making \\
Strategies with colleagues \\
1. Conflict management interventions \\
2. Emotional and experiential sharing of caring for dying patients \\
3. Collaboration with interdisciplinary team members
\end{tabular}

content analysis (the Innate, Individual, Relational and Societal Rings). However, as strands of the theme labeled 'interactions' involve aspects that move beyond the Societal Ring, an additional theme labeled 'conflicts in providing EoL (end of life) care' was created. As such, this saw the combined results and presentation of six themes/categories which correspond to the four rings of RToP, conflicts in providing EoL care and coping strategies.

\section{Comparisons between tabulated summaries, thematic analysis and directed content analysis}

Combined themes after the funneling process are presented in Table 3 (without references) and in Additional file 4 (with references). Given that many of the 'codes' within these category/ themes are poorly described, they have been tabulated to help analysis. To clarify, Category/ Theme 1 to 4 discuss general accounts of caring for dying patients in

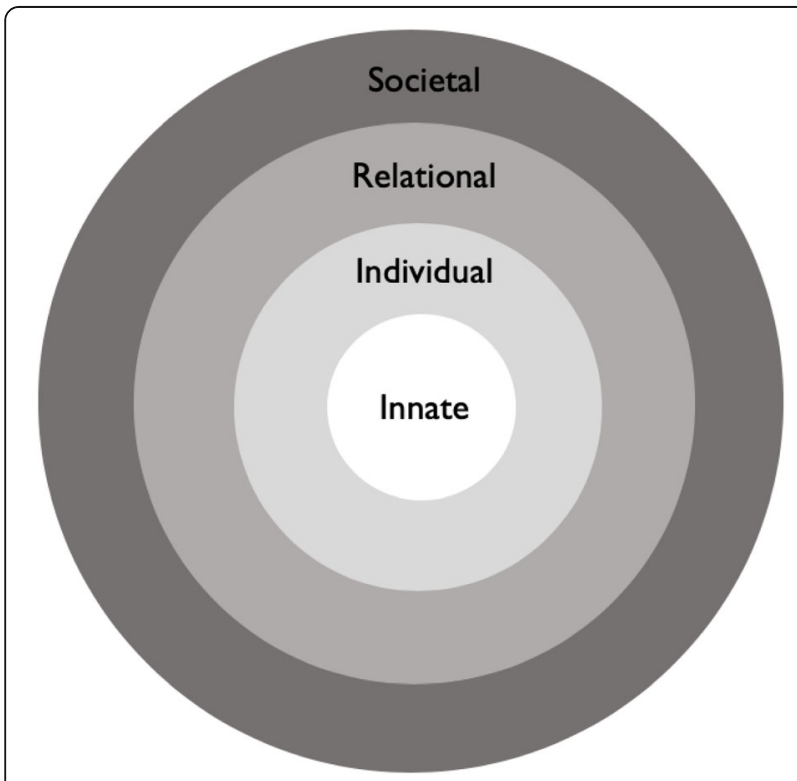

Fig. 3 Krishna and Alsuwaigh (2015)'s Ring Theory of Personhood
ICU whilst Category/ Theme 5 relates to specific accounts of experiences providing EoL care in ICU. Category/ Theme 6 review accounts of coping strategies adopted by ICU physicians.

Category/ theme 1: innate ring Thnces an ICU physician's views of their own mortality. It also includes their perspectives on whether or not they conceive the death of a patient as a failure. It includes the physician's beliefs on their duty of care at end of life, particularly in relation to alleviating suffering, ensuring a 'good death' and their views surrounding one's 'right to die'.

Category/ theme 2: individual ring This considers the physician's beliefs, values, principles, personality, abilities, thoughts, emotions and actions with respect to death and dying. It includes factors that affect their thinking and decision making about EoL care such as the need to manage their own expectations, concerns and doubts about prognostication; their role in determining overall goals of care; and their ability to communicate these decisions to colleagues, patients and their families whilst showing empathy.

Category/ theme 3: relational ring This considers the impact of the emotional and psychological self-doubt and apprehension they experience when caring for dying patients upon personal relationships the ICU physician deems important to them.

Category/ theme 4: societal ring This considers the influence of sociocultural norms and societal expectations of the physician's professional relationships and roles, and how they impact legal and professional standards. It includes the physical and practical issues affecting EoL care. It also considers the effect of caring for the dying on their relationships with colleagues, patients and their families. 
Table 3 Combined Categories/ Themes

\section{Subcategory}

Category/ Theme 1: Innate Ring

1. Perception of life and death

\section{Category/ Theme 2: Individual Ring}

1. Ability to make sense of things

2. Ability to communicate and relate

3. Ability to express feelings

4. Acquired ability

\section{Elaborations}
a) Confrontation with own mortality
b) Conception of a good death impacting end-of-life care
c) Death of patient perceived to be a personal failure
d) Death of patient not perceived to be a personal failure
e) Conflict about prolonging life as it prolonged suffering
f) One has a right to die

Impaired end-of-life decision making:
a) Personal factors
b) Patient factors
c) Institutional factors
d) Societal culture

Doubt:

e) Doubt in end-of-life decision making

f) Doubt about assessment of patient's prognosis

g) Doubt due to uncertainties in patient's trajectories

h) Internal conflict when balancing care goals

i) Internal conflict when managing own expectations

j) Dilemmas about active treatment versus palliative intention

a) Loss of ability to communicate and relate to patients

b) Poor communication skills

c) Difficulty and discomfort when broaching topic of death to patients

d) Attempts to avoid discussion of death in general

e) Improvement in communication skills

f) Confidence in ability to navigate difficult conversations

g) Motivated to further improve communication skills

a) Emotional detachment

b) Emotions perceived as hinderance to job

Apprehension/Distress:

c) From end-of-life care

d) From communication with family

e) From belief that futile treatment prolonged dying process

f) From possibility of litigation

g) Fear due to unintentional transference to own family

h) Emotional involvement being considered as valuable

i) Satisfaction in involvement in patient's end-of-life care
a) Lacking knowledge about end-of-life
b) Inadequate opportunities for end-of-life care training
c) Doubt and lack of confidence in clinical skills
d) Testing of practical skills such as treatment withdrawal techniques
e) End-of-life decision making differing with years of experience
f) Acquisition of new skills with experience
g) Adequate end-of-life care training 
Table 3 Combined Categories/ Themes (Continued)

Subcategory

5. Beliefs

6. Perceived Role as a doctor

\section{Category/ Theme 3: Relational Ring}

1. Family

\section{Category/ Theme 4: Societal Ring}

1. Physical environment

2. Cultural norms

3. Workplace cultural norms

4. Societal expectations

\section{Elaborations}

Personal Beliefs

a) Conflicting beliefs resulting in distress

b) Dilemmas about the balancing of opposing values

c) Personal beliefs reflected in end-of-life practices and communication Ethical dilemmas

d) Differences in ethical opinion surrounding treatment withholding and withdrawal

e) Futile treatment

f) Lack of advanced directives and families' aggressive care requests causing moral distress

Religious views

g) Influenced end-of-life discussion and decision making

h) Did not influence end-of-life practices

a) To care for dying patients

b) Perceived duty to prolong life causing moral distress

c) Death of patient perceived to be a professional failure

d) Death of patient not perceived to be a professional failure

e) Uncertainty about role in end-of-life discussions resulting in no/late end-of-life discussion

f) Paternalistic approach to decision making

g) Perceived professional duty to collaborate and care for needs of patient's family

h) Professional satisfaction from caring for dying patients

i) Satisfaction upon reconciling dual role of saving lives and managing death well

j) Emotions perceived as hinderance to role as doctor

a) Fear due to unintentional transference to physician's own family members

a) Availability of resources in different countries influencing end-of-life care

b) Intensive care unit environment as not conducive for end-of-life discussions

Intensive care unit as an inappropriate place to die

c) Lack of privacy

d) Focus of care not allowing for palliative care

Suitability for palliative care teaching

e) Not suitable

f) Suitable

a) Physician's end-of-life care attitudes, behaviors and decisions privy to cultural norms

b) Death and dying perceived as a "taboo" topic in certain cultures

c) Need for end-of-life care to be sensitive to different cultures encountered

a) Influencing views on death, end-of-life care attitudes, behavior and decision making

a) Societal expectations promoting survival and death prevention 
Table 3 Combined Categories/ Themes (Continued)

Subcategory

5. Legal standard

6. Professional Relationships

\section{Elaborations}

b) Perception of treatment withdrawal as taking the life of one's patient affecting physician's end-of-life decision making

a) Fear of legal challenge affecting end-of-life care leading to defensive practice

b) Unclear laws surrounding end-of-life practices breeding legal uncertainty

c) Adherence to decisions despite perceived potential legal kickback

Patients

a) Challenges faced during end-of-life communication

b) Managing expectations of patients

c) Inspiring interactions with patients

Patient's family

d) Conflict between physician and patient's family

e) Effects of conflict on relationship

f) Family members concerned for patient's possible pain and distress

g) Managing expectations of patient's family members

h) Family's distress after end-of-life care discussion

i) Empowering interactions with patient's family members

j) Factors affecting communication with family members

k) Creation of soft landing when informing family about death

I) Perception of intensive care unit as not conducive for palliative care discussions

Nurses \& intensive care unit team

m) Support from other intensive care unit physicians to help manage end-of-life decisions

Physicians from other specialties

n) Challenges with interaction

o) Lack of understanding of one another's role

a) Professional expectation for doctors to not cause death or harm to patients

b) Responsibility to decide on withdrawal of treatment went against physician's perceived professional standards

\section{Category/ Theme 5: Conflicts in providing end-of-life care}

1. Interpretation of duty of the physician

2.Behavior of the physician

3.Behavior of others

4.Professional Standards a) Professional expectation that doctors should not cause death or harm to patients

b) Responsibility of treatment withdrawal decision going against physician's perceived professional standards

c) Physician's end-of-life care attitudes, behaviors and decisions d) Need for end-of-life care to be sensitive to different cultured encountered

a) Doubts in self, conflicts in decision making

b) Emotional and psychological overlay

c) Internal conflict between beliefs and duties

e) Conflict with intensive care unit nurses

f) Challenges with interactions with other professionals

g) Perception that nurses do not grasp the complexity of end-of-life decision making

h) Conflict between respect for cultural norms and general practice i) Conflict between team members on how to interpret way to proceed in grey situations 
Table 3 Combined Categories/ Themes (Continued)

\begin{tabular}{|c|c|}
\hline Subcategory & Elaborations \\
\hline \multicolumn{2}{|c|}{ Category/ Theme 6: Coping strategies } \\
\hline 1. Personal strategies & $\begin{array}{l}\text { a) Effective communication to strengthen decision making position } \\
\text { Confidence: } \\
\text { b) Gaining confidence through experience } \\
\text { c) Gaining confidence with end-of-life discussions } \\
\text { d) Taking breaks from the intensive care unit or practicing on other sites }\end{array}$ \\
\hline 2. Strategies with patients & a) Collaboration with patient to reduce moral burden of decision making \\
\hline 3. Strategies with patient's family & $\begin{array}{l}\text { a) Creation of soft landing when informing patient's family about death } \\
\text { b) Collaboration with patient's family to reduce moral burden of } \\
\text { decision making }\end{array}$ \\
\hline 4. Strategies with colleagues & $\begin{array}{l}\text { a) Conflict management interventions } \\
\text { b) Emotional and experiential sharing of caring for dying patients } \\
\text { c) Collaborations with interdisciplinary team members }\end{array}$ \\
\hline
\end{tabular}

Category/ theme 5: conflicts in providing EoL care This moves beyond the general considerations discussed previously and regards specific accounts of internal conflicts affecting the physician, and conflicts with other healthcare professionals that arise in the provision of EoL care.

Category/theme 6: coping strategies These include general and specific accounts of coping strategies adopted by physicians providing EoL care in ICUs. These accounts are categorized as a distinct category /theme despite their multimodal approach involving personal, professional and existential factors and strategies involving collaboration and sharing with other professionals.

\section{Stage 5 of SEBA: discussion}

\section{Discussion}

This SSR reveals an intricate array of intimately entwined responses by ICU physicians as a result of caring for dying patients. Highlighted by the themes 'conflicts in providing EoL care' and 'coping strategies', this SSR suggests that it has diverse ramifications including complex immediate and long-term effects on the ICU physician's thinking, decision making and actions. Such change underscores the need to review how they are supported. The data rebuffs the notion that 'single point interventions' such as debrief sessions following a difficult case or a meeting with a psychologist or counsellor will sufficiently address the complex effects on their psycho-social, existential, personal and professional self-concepts. Rather this SSR will argue for a more longitudinal and holistic response to the support of ICU physicians that entails careful study of their individual concepts of personhood.

Contemplating these effects through the lens of the Ring Theory of Personhood (RToP) suggest that support should be directed at the beliefs, values and principles (Internal Constituents or IC) contained within the different rings of the ICU physician's personhood. These arise from spiritual beliefs, familial credos, cultural norms, societal values and personal philosophies adopted. These, however, may surface in more than one ring thus reiterating their connection or entwined nature.

With ICs overlapping and also influencing a physician's thinking, decision making and actions, ensuring that the ICs are in sync with one another is critical. For the rings to be in 'synchrony', these constituents must be congruous. For example, belief in the sanctity of life (Innate Ring) ought to be mirrored in how the physician perceives his role as a healthcare professional (Individual and Societal Ring) [30].

However, maintaining synchrony between the rings can be challenging particularly as ICU physicians face acute shortages of resources [31] and manpower, complex clinical cases [32] and unprecedented levels of death in ICUs. This is further aggrandized in times of national and global crisis [33]. These circumstances can create discordance within and between ICs through the creation of competing obligations. For example, when treatment options have been exhausted and continued ventilation of the ICU patient is deemed to be futile, the physician will be faced with the difficult choice of withdrawing ventilatory support and allowing the patient to die. This would run contrary to their belief in the sanctity of life [34, 35]. How the physician addresses this 'catalyst' will determine their actions.

A 'catalyst' is seen as a situation where a physician is forced to question their position and/or the ethical, moral, existential and personal beliefs, values and principles that they hold. If unaddressed a catalyst will result in 'conflict' within individual rings. If conflicts are not resolved effectively, they may result in further 'dyssynchrony' between rings as will be discussed later.

For now, this SSR suggests that the RToP provides a means of understanding the complex interplay of psychoemotional, existential and relational issues at hand. The 
RToP suggests that in making decisions as to whether to withdraw futile treatment, physicians face catalysts in the form of doubts surrounding their roles in the provision of EoL care, the alleviation of suffering and their patient's right to die [36, 37]. The moral, existential, personal and professional conflict between ICs in their Innate Ring is also made manifest in their Individual Ring as they face anxiety and doubts over their knowledge, skills and ability to provide effective and empathic care for these dying patients and their families as they meet their clinical obligations $[38,39]$.

These also impact their Relational Ring as they may compromise the quality and nature of personal relationships that the physician holds dear to them, thus threatening an important source of support as they grapple with these concerns [40]. Furthermore, struggles with balancing individual beliefs and values with societal, religious, cultural obligations and legal, professional, ethical standards of practice that stem from their Societal Ring serve to compound the ICU physician's coping strategies and ultimately impair their ability to provide efficacious care [41-43]. The presence of multiple rings in conflict suggest that catalysts may generate ripples and have bearings on more than one.

Persistent unresolved conflicts between rings - the aforementioned dyssynchrony - may result in acute or prolonged moral, spiritual, emotional and psychological distress [44-46]. This may bear grave repercussions on their functioning as empathetic, reliable and effective healthcare providers with vulnerable patients under their care [47].

Conflict resolution may see ICU physicians prioritize their overarching goals based on their particular situation, professional and clinical obligations, beliefs, values and principles contained within their ICs and in keeping with regnant legal, professional and ethical standards $[48,49]$. This process offers potential assurance and reprieve for the addled and overwhelmed physician. Yet in deciding to withdraw treatment, the physician's decision to prioritize their clinical and professional obligations over their tendencies to err on the side of preserving life by virtue of their ICs in order to save the life of another patient with a better prognosis and less co-morbidities does not necessarily resolve the conflicts within the rings.

Here reframing the situation is critical. This process of reframing is guided by due consideration of contextual factors given the futility of prevailing treatment, the lack of alternative treatment options, the continued deterioration of the patient and the patient's general poor prognosis [50-52]. Similarly, the physician may reframe the situation and acknowledge that it is also their duty to cease futile and potentially burdensome treatment to prevent suffering, avoid prolonging the dying phase or return the patient to the original disease trajectory after a trial of treatment [53-60]. This process of reframing is key to realigning ICs, attenuating conflicts within the rings and preventing or assuaging dyssynchrony between the rings. Resolution of individual conflicts must necessarily consider that the resolutions arrived at are not at odds with ICs in other rings so as to not rouse dyssynchrony.

Evidencing the need for a comprehensive response to conflicts is the ICU physician's employment of multipronged coping mechanisms which draw on all four rings. This response includes a combination of timely, longitudinal, holistic interprofessional team support, collaborative efforts at care provision, refresher courses to gain greater confidence in one's practice and time off/rotations out of the ICU as shown in Table 3. Resolving dyssynchrony helps to bring the rings into harmony or 'synchrony', facilitating the professional and personal growth of the physician [40].

Establishing 'synchrony' between the rings through use of prioritization and reframing also helps sustain the ICs against catalysts. This ability to bring about and sustain synchrony is seen as a sign of resilience.

\section{Resilience}

Resilience is built by the ability to blunt or repel challenges to ICs brought about by catalysts. These take the form of internal and external factors (henceforth buffers). Internal buffers include prior experiences with similar catalysts and the possession of ethical, existential, practical and clinical skills [61-63]. Perhaps the most significant external buffer is discussing issues frankly with colleagues and garnering insight [64-67]. Other external buffers include having time outside of the ICU and a nurturing work environment $[49,68]$. Buffers allow physicians "to enjoy a positive experience" caring for an end-of-life patient [37] despite the difficult circumstances and conflicts that may arise within their individual ICs.

Conversely 'concession' sees the individual in a state of prolonged dyssynchrony which manifests as negative emotional, psychological, and spiritual burden and in the long term is seen to result in burnout, disillusionment and exit from the specialty [69] (Fig. 4).

\section{Evolution of the ring structure}

Whilst Radha Krishna and Alsuwaigh [28]'s original concept of the RToP has served as a means of understanding the issues affecting ICU physicians, it could also prove useful as a means of guiding support for physicians.

To operationalize the RToP, a review of its evolution is required. Developing from rings whose size depended on the number of elements within them, recent incarnations of the RToP conceive the rings as cylinders where the importance of ICs, as determined by the person, adds depth to the rings (Fig. 5). The concept of 


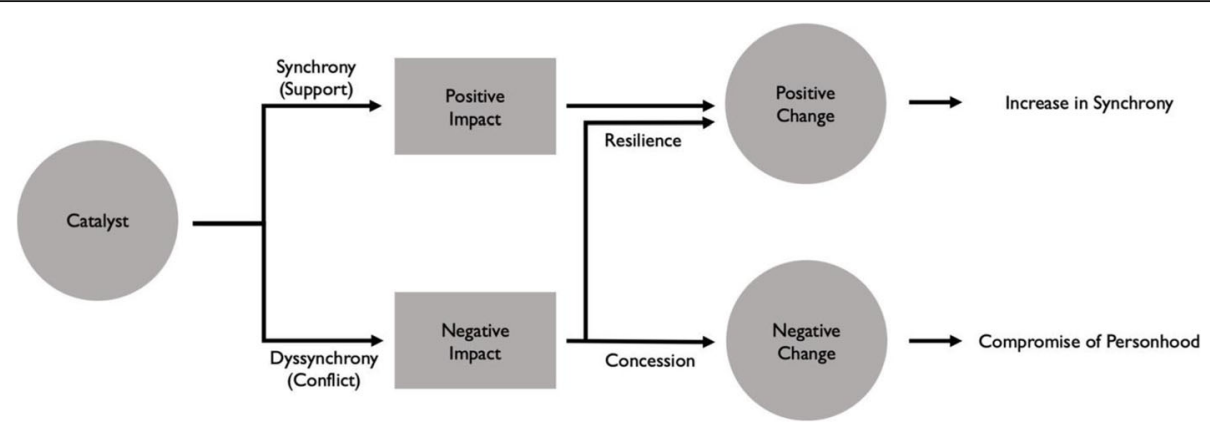

Fig. 4 Resilience and Concession

importance finds weight in this SSR and foregrounds the concept of prioritization and reframing. In keeping with this concept, the more important the element within the IC is in each ring, the closer it is to the inner aspect of the cylinder.

However, the data from this SSR adds a further dimension to this formulation. This third dimension comes from the impact of 'buffers' and the ability to maintain the rings in changing conditions. This dimension is consistent with the overarching goals of personhood which is to maintain the integrity of their individual concepts. The 3D perspective of the RToP serves as a reminder that concepts of personhood are highly personalized and involves input from the individual physician. Critically, the spheres may be employed as a means of holistically assessing the needs of ICU physicians in the care of dying patients.

Here the size of the spheres is determined by the number of elements within the ICs and the width or thickness corresponds to the element's importance arranged concentrically with the most important elements lying close to the core of this sphere. The depth of this sphere is afforded by the presence and quality of buffers. Here the quality of the buffers corresponds to their ability to repel catalysts and maintain the ICs. (Fig. 6).

\section{Limitations}

Although the search process was vetted and overseen by the expert team, use of specific search terms and inclusion of only English language articles potentiates the

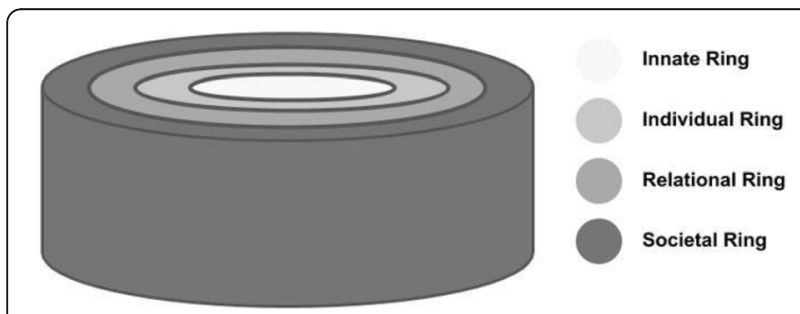

Fig. 5 Cylinder Model possibility of key publications being omitted. In addition, whilst independent and concurrent use of thematic and content analysis by the team of researchers improved its trustworthiness through enhanced triangulation and transparency, biases cannot be entirely eradicated.

Use of the RToP in this context is also novel and the data captured in this SSR suggests that its use as a 'tool' to identify critical issues with physicians' concepts of personhood still possesses limitations. Although the RToP provides a 'snapshot' of these prevailing concepts, the funneling process evidences the need for further studies to be conducted as to how personhood may evolve in both ordinary and extraordinary circumstances, and how physicians may address and cope with these diverse situational considerations. Finally, despite the presence of nursing data that echo the findings of this review, these findings may not truly reflect the unique challenges that ICU physicians experience, especially during pandemics such as COVID-19.

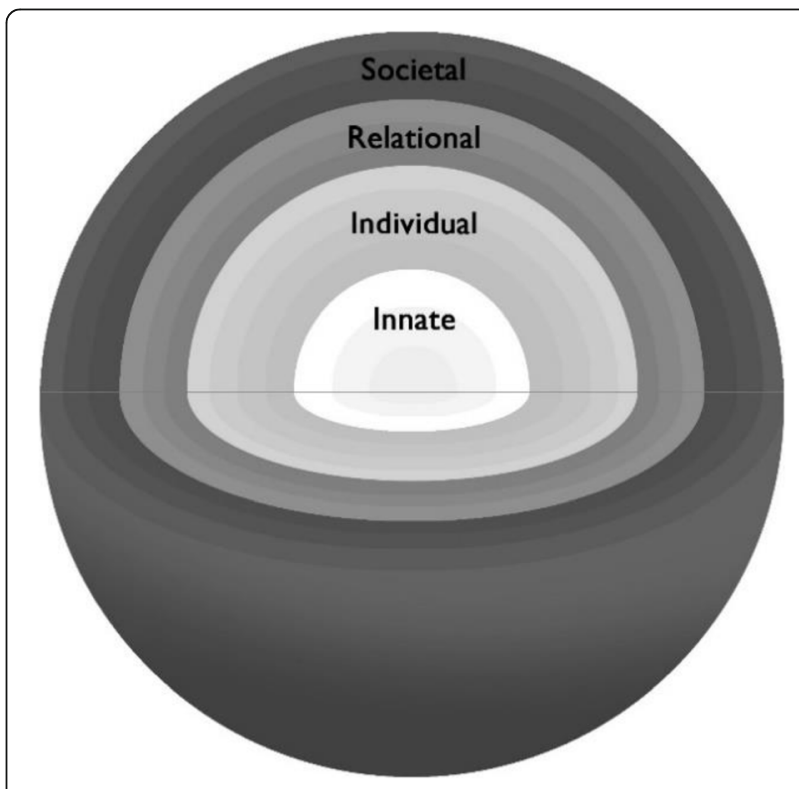

Fig. 6 The Sphere Model 


\section{Conclusion}

The COVID-19 pandemic has presented unprecedented challenges for ICU physicians facing death and dying. Data drawn from 'peace time' suggests that ICU physicians cope variably with different issues they face, underlining the need for personalized understanding of their particular situation and the presence of comprehensive long term support.

Indeed, the RToP lends itself to the design of such support structures with the first step calling for identification and recognition of physicians who are in a state of 'dyssynchrony' or 'concession'. Ethical guidance, intercollegial support and a nurturing work environment providing personalized debrief sessions and time out of the ICU will aid in reducing protracted dissonance between the beliefs, values and principles contained within each ring. This will naturally guide the physician towards 'synchrony' and alignment of these internal constituents - in short, the building of resilience.

As such, it is hoped that the four rings of the RToP will be carefully considered in the curation of longitudinal and holistic assessment tools used to discern the wide-ranging needs of overwhelmed ICU physicians. This is especially in the face of evidence that the impact of death and dying may ripple and implicate multiple rings of their personhood. It is only with assiduous analysis of all four rings that there can be greater certainty that all possible ramifications of death and dying are appropriately accounted for. These considerations if successfully attended to will certainly bolster the ICU physician.

Whilst these suggestions need further study, some of the lessons drawn are applicable to the COVID-19 pandemic where increased workload, manpower limitations, resource scarcity and complex triaging of vulnerable patients may see the amplification of moral and existential distress. Whilst resilience is crucial in ordinary 'peace time' as it wards against burnout, disillusionment and the phenomena which sees physicians leaving the specialty, it is especially necessary in the precarious climate of the COVID-19 era. With resilience not only do we protect the physician but we also protect the wider healthcare system.

\section{Supplementary information}

The online version contains supplementary material available at https://doi. org/10.1186/s13010-020-00096-1

Additional file 1. Pubmed Search Strategy. Search strategy employed as part of the systematic scoping review process. (DOCX $15.5 \mathrm{~kb}$ )

Additional file 2. Summary of included articles. Summaries of key points of articles included with MERSQI and COREQ quality assessment. (DOCX $131 \mathrm{~kb}$ )

Additional file 3. Summary of Thematic Analysis and Direct Content Analysis with References. Themes and categories identified in analysis with references. (DOCX $714 \mathrm{~kb}$ )

Additional file 4. Combined Themes with References. Combined themes after the funneling process with references. (DOCX $768 \mathrm{~kb}$ )

\section{Abbreviations}

ICU: Intensive care units; SSR: Systematic scoping review; SEBA: Systematic evidenced based approach; RToP: Ring theory of personhood; PCC: Population, context and concept; MERSQI: Medical education research study quality instrument; COREQ: Consolidated criteria for reporting qualitative studies; BEME: Best evidence medical education;

STORIES: structured approach to the reporting in healthcare education of evidence synthesis; EoL: End of life; IC: Internal constituent

\section{Acknowledgements}

The authors would like to dedicate this paper to the late Dr. S Radha Krishna whose advice and ideas were integral to the success of this study. The authors would like to thank the anonymous reviewers whose advice and feedback greatly improved this manuscript.

\section{Authors' contributions}

JTYK, LXLN, NHAK, JLC, NPXC, ABHMA, CYH, LKRK were involved in the conception and design of this study, data acquisition, analysis and interpretation of data and writing of the publication. JLG and MSQK have made contributions to the data acquisition. LHET, YTO, MC, LKRK have revised the work. AMCC, SM and LKRK have supervised the team. All authors read and approved the final manuscript.

Funding

No funding was received for this study.

\section{Availability of data and materials}

All data generated or analysed during this study are included in this published article.

Ethics approval and consent to participate

Not applicable.

Consent for publication

Not applicable.

\section{Competing interests}

All authors declare that there was no conflict of interest.

\section{Author details}

${ }^{1}$ Yong Loo Lin School of Medicine, National University of Singapore, Singapore, Singapore. ${ }^{2}$ Division of Supportive and Palliative Care, National Cancer Centre Singapore, Singapore, Singapore. ${ }^{3}$ Division of Cancer Education, National Cancer Centre Singapore, Singapore, Singapore. ${ }^{4}$ Medical Library, National University of Singapore Libraries, National University of Singapore, Singapore, Singapore. ${ }^{5}$ Palliative Care Institute Liverpool, Academic Palliative \& End of Life Care Centre, Cancer Research Centre, University of Liverpool, 200 London Road, Liverpool L3 9TA, UK. ${ }^{6}$ Duke-NUS Graduate Medical School, Singapore, Singapore. ${ }^{7}$ Centre for Biomedical Ethics, National University of Singapore, Singapore, Singapore. ${ }^{8} \mathrm{PalC}$, The Palliative Care Centre for Excellence in Research and Education, Singapore, Singapore.

Received: 5 June 2020 Accepted: 11 October 2020

Published online: 25 November 2020

References

1. Phua J, Weng L, Ling L, Egi M, Lim CM, Divatia JV, Shrestha BR, Arabi YM, Ng J, Gomersall CD, Nishimura M, Koh Y, Du B, Asian Critical Care Clinical Trials Group. Intensive care management of coronavirus disease 2019 (COVID-19): challenges and recommendations. Lancet Respir Med. 2020;8(5):506-17. https://doi.org/10.1016/S2213-2600(20)30161-2.

2. Wax RS, Christian MD. Practical recommendations for critical care and anesthesiology teams caring for novel coronavirus (2019-nCoV) patients. Can J Anaesth. 2020;67(5):568-76.

3. Sasangohar F, Jones SL, Masud FN, Vahidy FS, Kash BA. Provider burnout and fatigue during the COVID-19 pandemic: lessons learned from a highvolume intensive care unit. Anesth Analg. 2020.

4. Lai J, Ma S, Wang Y, Cai Z, Hu J, Wei N, et al. Factors associated with mental health outcomes among health care workers exposed to coronavirus disease 2019. JAMA Netw Open. 2020;3(3):e203976. 
5. Adams JG, Walls RM. Supporting the Health Care Workforce During the COVID-19 Global Epidemic. JAMA. 2020;323(15):1439-40. https://doi.org/10 1001/jama.2020.3972.

6. Shen X, Zou X, Zhong X, Yan J, Li L. Psychological stress of ICU nurses in the time of COVID-19. Crit Care. 2020;24(1):200.

7. Huang L, Lin G, Tang L, Yu L, Zhou Z. Special attention to nurses' protection during the COVID-19 epidemic. Crit Care. 2020;24(1):120.

8. Brooks SK, Webster RK, Smith LE, Woodland L, Wessely S, Greenberg N, et al. The psychological impact of quarantine and how to reduce it: rapid review of the evidence. Lancet. 2020;395(10227):912-20.

9. Bai Y, Lin CC, Lin CY, Chen JY, Chue CM, Chou P. Survey of stress reactions among health care workers involved with the SARS outbreak. Psychiatr Serv. 2004;55(9):1055-7.

10. Marjanovic Z, Greenglass ER, Coffey S. The relevance of psychosocial variables and working conditions in predicting nurses' coping strategies during the SARS crisis: an online questionnaire survey. Int I Nurs Stud. 2007; 44(6):991-8.

11. Liew MF, Siow WT, MacLaren G, See KC. Preparing for COVID-19: early experience from an intensive care unit in Singapore. Crit Care. 2020;24(1):83.

12. Cunningham K. COVID-19 Through a Nurse's Lens 2020 [Available from: https://www.newyorker.com/magazine/2020/05/04/a-city-nurse.

13. Pring R. The 'false dualism' of educational research. J Philos Educ. 2000;34(2): 247-60.

14. Crotty M. The foundations of social research: meaning and perspective in the research process. London: SAGE; 1998.

15. Ford DW, Downey L, Engelberg R, Back AL, Curtis JR. Discussing religion and spirituality is an advanced communication skill: an exploratory structural equation model of physician trainee self-ratings. J Palliat Med. 2012;15(1):63-70.

16. Schick-Makaroff K, MacDonald M, Plummer M, Burgess J, Neander W. What synthesis methodology should I use? A review and analysis of approaches to research synthesis. AlMS Public Health. 2016;3(1):172-215.

17. Peters $M$, Godfrey C, Mclnerney P, Soares $C$, Khali $H$, Parker D. The Joanna Briggs institute reviewers' manual 2015: methodology for JBI scoping reviews 2015 April 29, 2019. Available from: http://joannabriggs.org/assets/ docs/sumari/Reviewers-Manual_Methodology-for-JBI-Scoping-Reviews_2 015_v1.pdf.

18. Peters MD, Godfrey CM, Khalil H, Mclnerney P, Parker D, Soares CB. Guidance for conducting systematic scoping reviews. Int J Evid Based Healthc. 2015;13(3):141-6.

19. Sambunjak D, Straus SE, Marusic A. A systematic review of qualitative research on the meaning and characteristics of mentoring in academic medicine. J Gen Intern Med. 2010;25(1):72-8.

20. Wong G, Greenhalgh T, Westhorp G, Buckingham J, Pawson R. RAMESES publication standards: meta-narrative reviews. BMC Med. 2013;11:20

21. Popay J, Roberts H, Sowden A, Petticrew M, Arai L, Rodgers M, et al. Guidance on the conduct of narrative synthesis in systematic reviews: A product from the ESRC Methods Programme 2006.

22. Reed DA, Beckman TJ, Wright SM, Levine RB, Kern DE, Cook DA. Predictive validity evidence for medical education research study quality instrument scores: quality of submissions to JGIM's medical education special issue. J Gen Intern Med. 2008;23(7):903-7.

23. Tong A, Sainsbury P, Craig J. Consolidated criteria for reporting qualitative research (COREQ): a 32-item checklist for interviews and focus groups. Int J Qual Health Care. 2007:19(6):349-57.

24. Braun V, Clarke V. Using thematic analysis in psychology. Qual Res Psychol. 2006;3(2):77-101.

25. Hsieh H-F, Shannon SE. Three approaches to qualitative content analysis. Qual Health Res. 2005;15(9):1277-88.

26. Haig A, Dozier M. BEME guide no. 3: systematic searching for evidence in medical education - part 2: constructing searches. Med Teach. 2003;25:463-84.

27. Gordon M, Gibbs T. STORIES statement: publication standards for healthcare education evidence synthesis. BMC Med. 2014;12(1):143.

28. Radha Krishna LK, Alsuwaigh R. Understanding the fluid nature of personhood - the ring theory of personhood. Bioethics. 2015;29(3):171-81.

29. Elo S, Kyngäs $\mathrm{H}$. The qualitative content analysis process. J Adv Nurs. 2008; 62(1):107-15.

30. Aita K, Kai I. Physicians' psychosocial barriers to different modes of withdrawal of life support in critical care: a qualitative study in Japan. Soc Sci Med. 2010;70(4):616-22.
31. Ball CG, Navsaria P, Kirkpatrick AW, Vercler C, Dixon E, Zink J, et al. The impact of country and culture on end-of-life care for injured patients: results from an international survey. J Trauma Inj Infect Crit Care. 2010; 69(6):1323-34.

32. Baggs JG, Schmitt MH, Prendergast TJ, Norton SA, Sellers CR, Quinn JR, et al. Who is attending? End-of-life decision making in the intensive care unit. J Palliat Med. 2012;15(1):56-62.

33. Joynt GM, Lipman J, Hartog C, Guidet B, Paruk F, Feldman C, et al. The Durban world congress ethics round table IV: health care professional endof-life decision making. J Crit Care. 2015;30(2):224-30.

34. Beck S, Loo A, Reiter-Theil S. A "little bit illegal"? Withholding and withdrawing of mechanical ventilation in the eyes of German intensive care physicians. Med Health Care Philos. 2008;11(1):7-16.

35. Hamric AB, Blackhall $L$ J. Nurse-physician perspectives on the care of dying patients in intensive care units: collaboration, moral distress, and ethical climate. Crit Care Med. 2007;35(2):422-9.

36. Çobanoglu N, Algier L. A qualitative analysis of ethical problems experienced by physicians and nurses in intensive care units in Turkey. Nurs Ethics. 2004;11(5):444-58.

37. Laurent A, Bonnet M, Capellier G, Aslanian P, Hebert P. Emotional impact of end-of-life decisions on professional relationships in the ICU: an obstacle to collegiality? Crit Care Med. 2017;45(12):2023-30.

38. Mehter HM, McCannon JB, Clark JA, Wiener RS. Physician approaches to conflict with families surrounding end-of-life decision-making in the intensive care unit. A qualitative study. Ann Am Thorac Soc. 2018;15(2):241-9.

39. Barnett MD, Williams BR, Tucker RO. Sudden advanced illness: an emerging concept among palliative care and surgical critical care physicians. Am J Hosp Palliat Care. 2016;33(4):321-6.

40. Wåhlin I, Ek A-C, Idvall E. Staff empowerment in intensive care: nurses' and physicians' lived experiences. Intensive Cri Care Nurs. 2010;26(5):262-9.

41. Chikhladze N, Janberidze E, Velijanashvili M, Chkhartishvili N, Jintcharadze M, Verne J, et al. Mismatch between physicians and family members views on communications about patients with chronic incurable diseases receiving care in critical and intensive care settings in Georgia: a quantitative observational survey. BMC Palliat Care. 2016;15:1-10.

42. Henrich NJ, Dodek PM, Alden L, Keenan SP, Reynolds S, Rodney P. Causes of moral distress in the intensive care unit: a qualitative study. J Crit Care. 2016;35:57-62.

43. Weng L, Joynt GM, Lee A, Du B, Leung P, Peng J, et al. Attitudes towards ethical problems in critical care medicine: the Chinese perspective. Intensive Care Med. 2011;37(4):655-64

44. Donnelly SM, Psirides A. Relatives' and staff's experience of patients dying in ICU. QJM. 2015;108(12):935-42.

45. McAndrew NS, Leske JS. A balancing act: experiences of nurses and physicians when making end-of-life decisions in intensive care units. Clin Nurs Res. 2015;24(4):357-74.

46. Workman S, McKeever P, Harvey W, Singer PA. Intensive care nurses' and physicians' experiences with demands for treatment: some implications for clinical practice. J Crit Care. 2003:18(1):17-21.

47. Teixeira C, Ribeiro O, Fonseca AM, Carvalho AS. Ethical decision making in intensive care units: a burnout risk factor? Results from a multicentre study conducted with physicians and nurses. J Med Ethics. 2014;40(2):97-103.

48. Brooks LA, Manias E, Nicholson P. Barriers, enablers and challenges to initiating end-of-life care in an Australian intensive care unit context. Aust Crit Care. 2017:30(3):161-6.

49. Robertsen A, Helseth E, Laake JH, Førde R. Neurocritical care physicians' doubt about whether to withdraw life-sustaining treatment the first days after devastating brain injury: an interview study. Scand J Trauma Resusc Emerg Med. 2019:27(1):81.

50. Daly BJ, Douglas SL, O'Toole E, Rowbottom J, Hoffer A, Lipson AR, et al. Complexity analysis of decision-making in the critically ill. J Intensive Care Med. 2018;33(10):557-66.

51. Park SY, Phua J, Nishimura M, Deng Y, Kang Y, Tada K, et al. End-of-life care in ICUs in East Asia: a comparison among China, Korea, and Japan. Crit Care Med. 2018;46(7):1114-24

52. Svantesson M, Sjökvist $P$, Thorsén $H$. End-of-life decisions in Swedish ICUs. How do physicians from the admitting department reason? Intensive Crit care Nurs. 2003;19(4):241-51.

53. Cameron J, Savulescu J, Wilkinson D. Is withdrawing treatment really more problematic than withholding treatment? Journal of medical ethics. 2020. 
54. English V, Mussell R, Sheather J, Sommerville A. Withdrawing artificial ventilation. J Med Ethics. 2006;32(8):495.

55. Foster $C$. It is never lawful or ethical to withdraw life-sustaining treatment from patients with prolonged disorders of consciousness. J Med Ethics. 2019:45(4):265-70.

56. Sulmasy DP, Sugarman J. t. J Med Ethics. 1994;20(4):218-22 discussion 23-4.

57. Vincent JL. Withdrawing may be preferable to withholding. Crit Care. 2005; 9(3):226-9.

58. Gatta B, Turnbull J. Providing palliative Care in the Medical ICU: a qualitative study of MICU Physicians' beliefs and practices. Am J Hosp Palliat Med. 2018:35(10):1309-13.

59. Trankle SA. Is a good death possible in Australian critical and acute settings?: physician experiences with end-of-life care. BMC Palliat Care. 2014 13:41. https://doi.org/10.1186/1472-684X-13-41.

60. Monteiro MC, Magalhaes AS, Feres-Carneiro T, Machado RN. Terminality in the ICU: the emotional and ethical dimensions of the medical care of the intensivist. Psicol Em Estud. 2016;21(1):65-75.

61. Brooks LA, Manias E, Nicholson P. Communication and decision-making about end-of-life Care in the Intensive Care Unit. Am J Crit Care. 2017;26(4): 336-41.

62. Festic E, Wilson ME, Gajic O, Divertie GD, Rabatin JT. Perspectives of physicians and nurses regarding end-of-life care in the intensive care unit. J Intensive Care Med. 2012;27(1):45-54.

63. Walter SD, Cook DJ, Guyatt GH, Spanier A, Jaeschke R, Todd TR, et al. Confidence in life-support decisions in the intensive care unit: a survey of healthcare workers. Canadian critical care trials group. Crit Care Med. 1998; 26(1):44-9.

64. Ferrand E, Lemaire F, Regnier B, Kuteifan K, Badet M, Asfar P, et al. Discrepancies between perceptions by physicians and nursing staff of intensive care unit end-of-life decisions. Am J Respir Crit Care Med. 2003; 167(10):1310-5.

65. Hough CL, Hudson LD, Salud A, Lahey T, Curtis JR. Death rounds: end-of-life discussions among medical residents in the intensive care unit. J Crit Care. 2005;20(1):20-5.

66. Jensen HI, Ammentorp J, Johannessen $\mathrm{H}$, Ørding $\mathrm{H}$. Challenges in end-oflife decisions in the intensive care unit: an ethical perspective. J Bioeth Inq. 2013;10(1):93-101.

67. Nordgren L, Olsson H. Palliative care in a coronary care unit: a qualitative study of physicians' and nurses' perceptions. J Clin Nurs. 2004;13(2):185-93.

68. Gutierrez KM. Advance directives in an intensive care unit: experiences and recommendations of critical care nurses and physicians. Critic Care Nurs Q. 2012;35(4):396-409.

69. Zambrano SC, Chur-Hansen A, Crawford GB. On the emotional connection of medical specialists dealing with death and dying: a qualitative study of oncologists, surgeons, intensive care specialists and palliative medicine specialists. BMJ Support Palliat Care. 2012;2(3):270-5.

\section{Publisher's Note}

Springer Nature remains neutral with regard to jurisdictional claims in published maps and institutional affiliations.

Ready to submit your research? Choose BMC and benefit from:

- fast, convenient online submission

- thorough peer review by experienced researchers in your field

- rapid publication on acceptance

- support for research data, including large and complex data types

- gold Open Access which fosters wider collaboration and increased citations

- maximum visibility for your research: over $100 \mathrm{M}$ website views per year

At $\mathrm{BMC}$, research is always in progress.

Learn more biomedcentral.com/submissions 